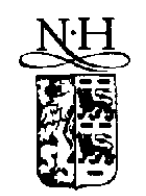

ELSEVIER

Applied Surface Science 78 (1994) 63-69

\title{
Surface structure and composition of high-surface-area molybdenum nitrides
}

\author{
B.G. Demczyk ${ }^{\text {a }}$, J.-G. Choi ${ }^{\text {b }}$, L.T. Thompson ${ }^{\text {b,* }}$ \\ ${ }^{a}$ Electron Microbeam Analysus Laboratory, Department of Materals Science and Enguneering, The University of Michigan, \\ Ann Arbor, MI 48109-2136, USA \\ ${ }^{b}$ Department of Chemical Engineering, The University of Michigan, Ann Arbor, MI 48109-2136, USA
}

(Recelved 20 September 1993; accepted for publication 11 December 1993)

\begin{abstract}
The determination of surface structure is critical in elucidating structure-function relationships for catalytic materials. However, such materials often consist of active regions of very limited spatial extent, rendering conventional bulk structural characterization techniques of limited utility. In this work, we have employed high-resolution transmission electron microscopy coupled with Fourier analysis, and X-ray photoelectron spectroscopy to determine the near-surface structures and compositions of a series of molybdenum nitride catalysts. The results show the near-surface to differ both in crystal structure and composition from the bulk. The bulk structure was $\gamma-\mathrm{Mo}_{2} \mathrm{~N}$ (fcc) while the lattice structure ncar the surface was body-centered. Many of the materials contained nitrogen in excess of that expected for phases in the Mo-N phase diagram. As the amount of nitrogen decreased, the oxygen content increased, and the calculated lattice parameter increased. Taken together, the results suggested the presence of $\mathrm{Mo}_{2} \mathrm{~N}_{3-x} \mathrm{O}_{x}$, a hypothetical primitive cubic structure, near the surface. This structure, which would produce a diffraction pattern that approximates that of a body-centered lattice, accounts for the near-surface structural and compositional properties of the high-surface-area molybdenum nitrides.
\end{abstract}

\section{Introduction}

The preparation of high-surface-area metal nitrides and carbides has sparked interest in their development as catalysts and/or catalyst supports. Molybdenum nitrides in particular are attractive due to their competitive activities for reactions including hydrodenitrogenation (Sajkowski and Oyama [1], Oyama et al. [2]) and $\mathrm{NH}_{3}$ synthesis (Volpe and Boudart [3]). The active

\footnotetext{
* Corresponding author Tel.: (313) 936-2015; Fax: (313) 7630459 .
}

sites for these catalytic reactions may consist of single atoms or groups of atoms near the surface (Sachtler [4]). While there is some information concerning the effects of bulk composition on the catalytic properties of these materials, there is currently very little known about the effects of surface structure and stoichiometry. In this work, we employed high-resolution transmission electron microscopy (HREM) and X-ray photoelectron spectroscopy (XPS) to evaluate the surface structures and compositions of a series of molybdenum nitride hydrodenitrogenation catalysts.

In high-resolution transmission electron microscopy, an atomic-scale image of a periodic 
specimen is produced as a result of the interaction of the incident electron beam with the periodic potential of the sample. Since the atomic structure contrast is lost (due to absorption) when the thickness of the material under examination exceeds 5 to $10 \mathrm{~nm}$, the technique provides a means of sampling the near-surface regions. As described in standard texts (Cowley [5]), there exists an inverse Fourier transform relationship between the atomic structure image and the electron diffraction pattern from which it is derived. By applying mathematical techniques such as fast Fourier transforms to selected areas of a suitably digitized image, diffraction information from very localized $(<5 \mathrm{~nm})$ regions may be obtained. Therefore, the technique affords very high spatial resolution phase analysis without the drawbacks of alternative small electron probe techniques (e.g. specimen contamination and motion during analysis). The limitation of the technique is that the material of interest must be thin enough to permit structure imaging $(\leq 10 \mathrm{~nm})$. Since most catalytic reactions occur at or near the free surface, the technique is ideal for elucidating nearsurface structures in catalytic materials. We have utilized this method to investigate the surfaces of molybdenum nitride catalysts, with particular attention focused on differences between the surface and bulk structures. X-ray photoelectron spectroscopy was used to determine the surface compositions of the materials before and after reduction in $\mathrm{H}_{2}$ using an in-situ reactor. Compositions determined by XPS were compared with bulk compositions derived from $\mathrm{X}$-ray diffraction (XRD).

\section{Experimental details}

Details concerning the synthesis of the molybdenum nitrides have been described elsewhere (Choi et al. [6]). In summary, we used the temperature-programmed reaction of $\mathrm{MoO}_{3}$ with $\mathrm{NH}_{3}$ to prepare a series of Mo nitrides with BET surface areas ranging from 4 to $116 \mathrm{~m}^{2} / \mathrm{g}$. Following synthesis, the materials were passivated during exposure to $1 \% \mathrm{O}_{2}$ in $\mathrm{He}$. This treatment prohibited bulk oxidation of the material. These materials were active and selective for pyridine hydrodenitrogenation.

Samples for HREM were prepared by gently grinding and ultrasonically dispersing the molybdenum nitride powders in isopropanol. The resulting suspension was then dropped onto holey carbon coated $3 \mathrm{~mm}$ diameter $\mathrm{Cu} 600$ mesh grids via a pipette. High-resolution transmission electron microscopy was performed at $400 \mathrm{kV}$, using a JEOL 4000 EX transmission electron microscope, which has a point-to-point resolution of $\leq 0.175 \mathrm{~nm}$. Subsequent analysis included digitizing the resulting film negatives into version 1.35 of NIH Image (Rasband [7]), with a Cohu Series $4810 \mathrm{CCD}$ camera. Version 1.35 was modified to incorporate a Fast Hartley Transform (hereafter referred to as FFT) routine for diffractogram analysis (Reeves [8]). The Hartley transform, being inherently real valued, provides advantages of speed and efficiency while retaining the salient features of the Fourier transform. Interplanar spacings were calculated with reference to a HREM image of silicon. Materials examined by HREM were in the passivated state.

Samples for X-ray photoelectron spectroscopic analysis were prepared by pressing $\sim 200 \mathrm{mg}$ of the Mo nitride powder into a self-supporting wafer. The spectroscopy was carried out using a Perkin-Elmer Phi 5400 spectrometer and an $\mathrm{Mg} \mathrm{K} \alpha$ X-ray source. Spectra of the materials were collected before and after reduction in flowing $\mathrm{H}_{2}$ (99.99\%, Air Products) at $673 \mathrm{~K}$ for $3 \mathrm{~h}$ using an in-situ reactor. The materials were reduced in an attempt to remove the surface passivation layer. Vacuum in the test chamber during transfer of the catalyst from the in-situ reactor and during the collection of spectra was typically better than $3 \times 10^{-9}$ Torr. The spectrometer energies were calibrated using the $\mathrm{Au}\left(4 \mathrm{f}_{1 / 2}\right)$ peak at $84.0 \mathrm{eV}$ and the $\mathrm{Cu}\left(2 \mathrm{p}_{3 / 2}\right)$ peak at $932.6 \mathrm{eV}$. Charging effects were accounted for by referencing the binding energies to that of adventitious carbon $(284.8 \mathrm{eV})$. Gaussian and/or Lorentzian peaks were deconvoluted using a non-linear least-squares fitting algorithm. The atomic compositions were estimated based on comparisons of the integrated peak intensities normalized by the atomic sensitivity factors. The atomic sensitiv- 
ity factors for the $\mathrm{Mo}\left(3 \mathrm{~d}_{5 / 2}\right), \operatorname{Mo}\left(3 \mathrm{p}_{3 / 2}\right), \mathrm{N}(1 \mathrm{~s})$, and $O(1 \mathrm{~s})$ peaks were $1.66,1.17,0.42$, and 0.66 , respectively (Briggs and Seah [9]). Errors in the composition were expected to be less than $\pm 15 \%$.

\section{Results}

\subsection{High-resolution electron microscopy}

Fig. 1 shows an experimental HREM image taken from MoN-2. The nearly square (four-fold rotation symmetry) arrangement of atomic columns strongly suggests a cubic structure. The inset shows the corresponding optical diffraction pattern (FFTs taken from digitized micrographs were identical), which also appeared as a square array of diffraction maxima. If we take the zone axis to be a $\langle 100\rangle$ cubic type, it is possible to assign indices to the principal reflections (see caption for Fig. 1) without a-priori knowledge of the lattice parameter. Such a diffraction pattern can arise in cubic or noncubic (with $c / a$ ratios significantly different from unity) structures. Here, $c$ and $a$ are unit-cell lattice parameters. However, in the noncubic systems, the zone axis would have to be a high-index one. Generally, we opted for the most basic interpretation, a cubic structure. Lattice parameters, obtained from FFTs of selected sections of the digitized HREM images were determined based on assumed facecentered or body-centered (nearly cubic) unit cells, or hexagonal structures. The zone axes chosen for analysis were the most unambiguous ones available: for square arrays we used the $\langle 100\rangle$ cubic type and for six-fold arrays, the [0001] hexagonal or $\langle 111\rangle$ cubic types. The bodycentered structures were found to be the most probable since they were more self-consistent with respect to both the [001] and [111] zone axes, that is, lattice parameters derived from the four-fold diffraction patterns agreed most closely with those obtained from the six-fold ones. Fitting the sixfold patterns to a hexagonal structure required a lattice parameter on the order of $0.3 \mathrm{~nm}$, which was deemed physically unrealistic.

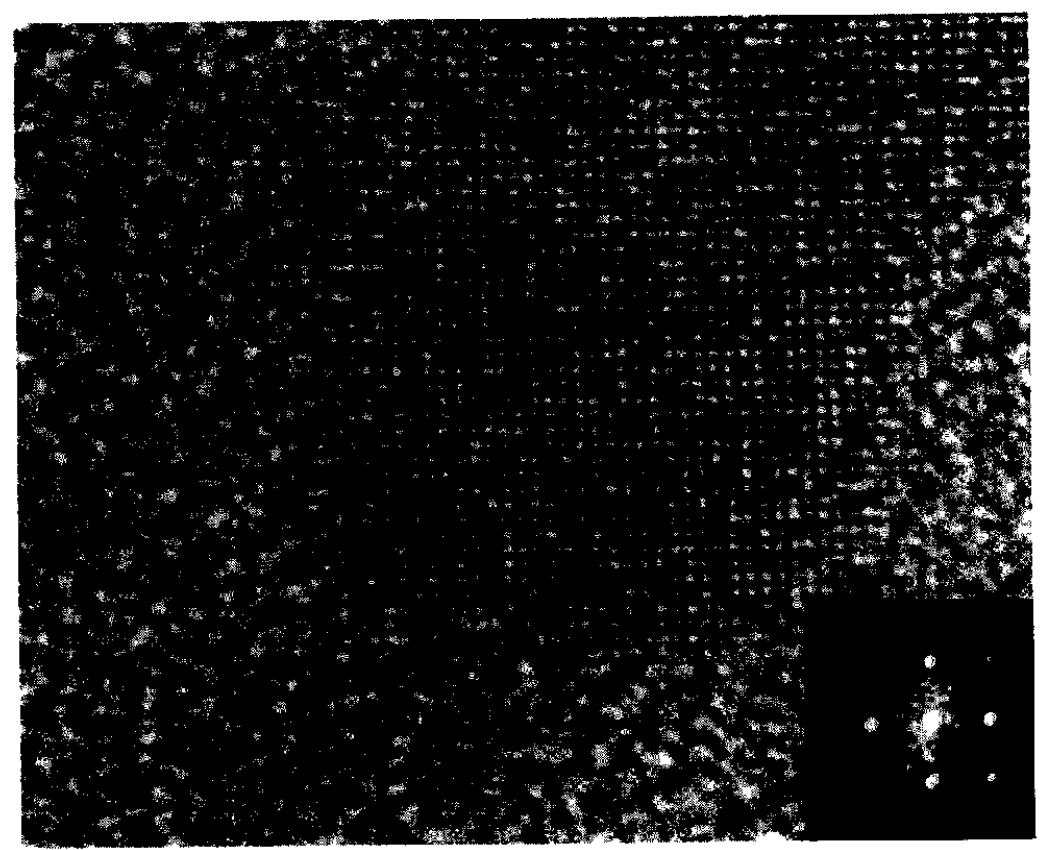

Fig. 1. Atomic-resolution transmission electron micrograph of MoN-2 lookıng down a $\langle 100\rangle$ zone axis, showing (001) and \{011\} atomic columns. The inset shows the corresponding optical diffraction pattern, containing (001) and (011) type reflections. 
Table 1 shows the experimentally determined lattice parameters. Since the structure images (and consequently the FFTs) represent a two-dimensional projection of the three-dimensional real-space structure, we could only assign two lattice parameters at a time (i.e. $c$ and $a$ ). There may well be distortions along the third axis (i.e. $b$ ), as well. It is quite possible that the surface consists of a distribution of distorted cubic (tetragonal or orthorhombic) structures with varying $c / a$ (and or $c / b$ or $b / a$ ) ratios. In all cases, however, the derived lattice parameters differed from that of $\gamma-\mathrm{Mo}_{2} \mathrm{~N}(a=0.416 \mathrm{~nm})$, which was the predominant phase observed from $\mathrm{X}$-ray diffraction analyses (Choi et al. [6]). Since the structure image (Fig. 1), by definition, arises from regions within $\leq 10 \mathrm{~nm}$ of the free surface of a particle, these results suggested that the nearsurface structure differed from that observed in the bulk. It should be noted that in general, interpretation of image detail and diffraction information obtained from a region comprised of a bulk phase plus an overlaying surface layer is not always straightforward (Yagi [10]). However, in the present case, following our definition of "near-surface" structure as that region from which the structure image arises, the bulk phase, as sampled by X-ray diffraction, does not contribute to the observed image. Therefore, there is no ambiguity in the interpretation of the experimental structure images and we attribute our observed HREM images to the near-surface region alone.

Table 1

Experımentally determined lattıce parameters $( \pm 5 \%)$

\begin{tabular}{llll}
\hline Sample & $a_{\mathrm{fc}}(\mathrm{nm})^{\mathrm{a}}$ & $a_{\mathrm{bc}}(\mathrm{nm})^{b}$ & $c / a$ \\
\hline MoN-1 & 0476 & 0.337 & 109 \\
MoN-2 & 0549 & 0.388 & 1.12 \\
MoN-3 & 0528 & 0.373 & 1.15 \\
MoN-4 & 0.432 & 0.305 & 1.02 \\
MoN-5 & 0.485 & 0.343 & 1.02 \\
MoN-6 & 0.447 & 0.316 & 1.02 \\
MoN-7 & 0.461 & 0.326 & 1.02 \\
MoN-8 & 0.496 & 0.351 & 1.14
\end{tabular}

${ }^{\mathrm{a}} a_{\mathrm{fc}}$ : lattice parameters based on assumed face-centered lattice.

${ }^{\mathrm{b}} a_{\mathrm{bc}}$. lattice parameters based on assumed body-centered lattice.
Table 2

Relative nitrogen and oxygen contents for the passivated and reduced materials

\begin{tabular}{|c|c|c|c|c|c|c|}
\hline \multirow{2}{*}{$\begin{array}{l}\text { Sample } \\
\text { No. }\end{array}$} & \multicolumn{3}{|c|}{ Passivated } & \multicolumn{3}{|c|}{ Reduced $^{a}$} \\
\hline & $\begin{array}{l}\text { N/ } \\
\text { Mo }\end{array}$ & $\begin{array}{l}\text { O/ } \\
\text { Mo }\end{array}$ & $\begin{array}{l}(\mathrm{N}+\mathrm{O}) / \\
\mathrm{Mo}\end{array}$ & $\begin{array}{l}\mathrm{N} / \\
\mathrm{Mo}\end{array}$ & $\begin{array}{l}\text { O/ } \\
\text { Mo }\end{array}$ & $\begin{array}{l}(\mathrm{N}+\mathrm{O}) / \\
\mathrm{Mo}\end{array}$ \\
\hline $\mathrm{MoN}-1$ & 104 & 0.69 & 1.73 & 0.48 & 0.18 & 0.66 \\
\hline MoN-2 & 1.14 & 0.84 & 1.98 & 0.60 & 0.20 & 0.80 \\
\hline MoN-3 & 1.45 & 1.15 & 260 & 0.93 & 0.24 & 1.17 \\
\hline $\mathrm{MoN}-4$ & 132 & 0.79 & 2.11 & 0.63 & 0.11 & 0.74 \\
\hline MoN-5 & 1.84 & 1.09 & 2.93 & 1.11 & 0.14 & 1.25 \\
\hline MoN-6 & 1.91 & 0.96 & 2.87 & 1.22 & 0.10 & 1.32 \\
\hline $\mathrm{MoN}-7$ & 094 & 0.84 & 178 & 1.00 & 0.16 & 1.16 \\
\hline MoN-8 & 0.92 & 1.07 & 1.99 & 0.91 & 0.13 & 1.04 \\
\hline
\end{tabular}

${ }^{a}$ Materials were reduced in an in-situ reactor at $673 \mathrm{~K}$ in flowing $\mathrm{H}_{2}$.

Distinct classes of $c / a$ ratios (1-1.02 and $1.09-1.15)$ were found in a number of the materials. We feel that these distortions from a cubic structure were related to the amount of nitrogen or oxygen in the lattice in regions near the surface. This possibility was investigated further by $\mathrm{X}$-ray photoelectron spectroscopy.

\subsection{X-ray photoelectron spectroscopy}

The nitrogen and oxygen contents for the asprepared and $\mathrm{H}_{2}$-reduced materials are given (relative to the Mo content) in Table 2. As these values were determined from the X-ray photoelectron spectra, they represent the near-surface composition (within 2-3 nm of the free surface). Two classes of materials emerged with respect to the sums of their $\mathrm{N} / \mathrm{Mo}$ and $\mathrm{O} / \mathrm{Mo}$ ratios. Asprepared materials with $(\mathrm{N}+\mathrm{O}) / \mathrm{Mo}$ ratios near 2.8 on reduction led to materials with $(\mathrm{N}+$ $\mathrm{O})$ /Mo ratios of $\sim 1.2$. These will be referred to as high-nitrogen-content materials, while the others will be referred to as low-nitrogen-content materials. As-prepared materials with ratios near 1.9, following reduction had $(\mathrm{N}+\mathrm{O}) /$ Mo ratios of $\sim 0.73$. The results indicated a connection between the compositions before and after reduction; therefore, compositional and structural information derived from the as-prepared materials should be useful in understanding the catalytic properties of the Mo nitrides. Since the mobilities 


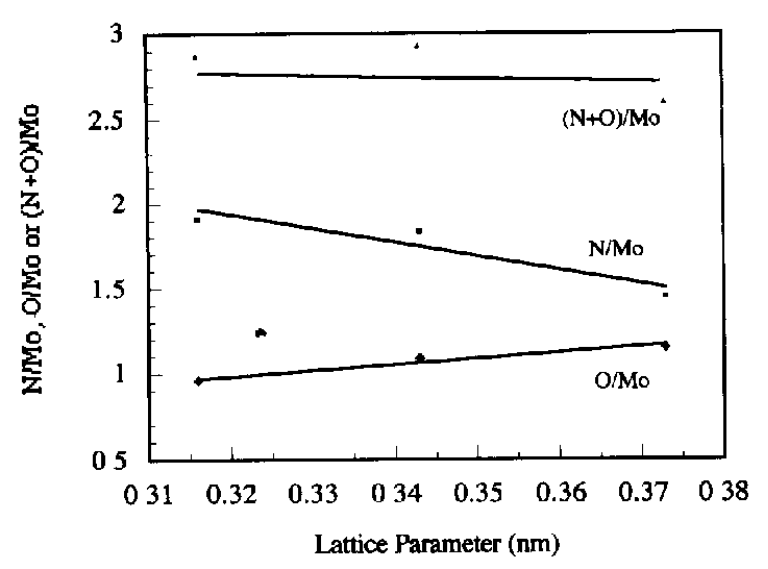

Fig. 2. $\mathrm{N} / \mathrm{Mo}, \mathrm{O} / \mathrm{Mo}$ and $(\mathrm{N}+\mathrm{O}) / \mathrm{Mo}$ ratios as a function of the calculated lattice parameter.

of nitrogen and oxygen are low at the reduction temperature $(673 \mathrm{~K})$, we did not expect reduction to significantly alter the structures of the materials.

In general, the nitrogen content decreased while the oxygen content increased as the lattice parameter estimated for the as-prepared and reduced materials increased. This response was most dramatic for the high-nitrogen-content materials (see Fig. 2). Note that the sums of the $\mathrm{N} / \mathrm{Mo}$ and $\mathrm{O} / \mathrm{Mo}$ ratios were approximately constant, indicating that nitrogen and oxygen occupied the same sites. These results also suggested that the radius of oxygen in the lattice was

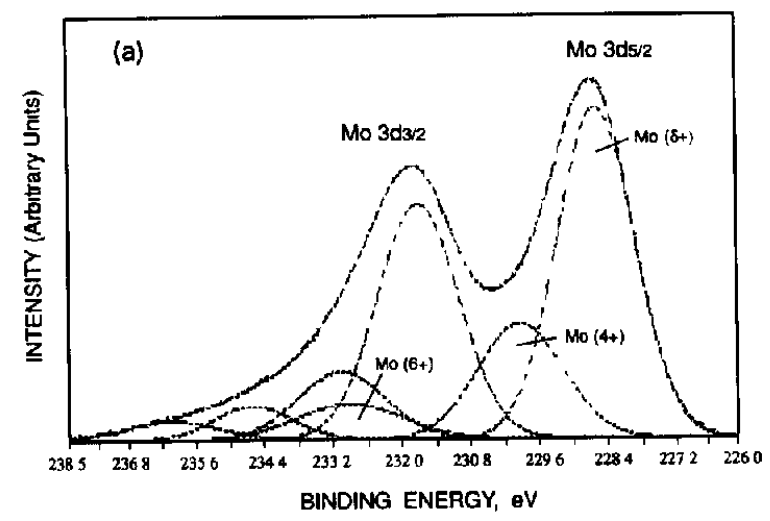

larger than that of nitrogen. While the atomic radius of oxygen is smaller than that of nitrogen, it is likely that both were present as ions in the lattice since the molybdenum was oxidized (see Fig. 3). There are several scenarios in which oxygen ions could be larger than nitrogen ions.

In a separate study we found that significant amounts of $\mathrm{NH}_{3}$, residue from the synthesis of the Mo nitrides, and $\mathrm{H}_{2} \mathrm{O}$ were adsorbed on the as-prepared materials (Choi et al. [11]); therefore, we were not surprised by the $(\mathrm{N}+\mathrm{O}) / \mathrm{Mo}$ ratios in excess of unity. The adsorbed $\mathrm{NH}_{3}$ and $\mathrm{H}_{2} \mathrm{O}$ were removed by heating the materials to $\sim 550$ $\mathrm{K}$ in $\mathrm{Ar}$ or $\mathrm{H}_{2}$. Small amounts of $\mathrm{H}_{2} \mathrm{O}$, most likely from the passivation layer; were also produced during high-temperature ( $>600 \mathrm{~K}$ ) reduction in $\mathrm{H}_{2}$. Little or no $\mathrm{NH}_{3}$ or $\mathrm{H}_{2} \mathrm{O}$ was removed at high temperature, suggesting that nitrogen left after reduction was the only nitrogen in the lattices of the as-prepared materials. For some of the Mo nitrides, the $\mathrm{N} / \mathrm{Mo}$ ratios for the as-prepared and reduced materials were virtually identical.

The nitrogen stoichiometries for the reduced materials were, for the most part, bracketed by those expected for the equilibrium Mo nitride phases. Nitrogen-to-molybdenum ratios for the low-nitrogen-content materials ranged from 0.48 to 0.63 , while the $\mathrm{N} / \mathrm{Mo}$ ratios for the highnitrogen-content materials were 0.93 to 1.22 . The nominal stoichiometries for $\beta-\mathrm{Mo}_{16} \mathrm{~N}_{7}, \gamma-\mathrm{Mo}_{2} \mathrm{~N}$ and $\delta$-MoN, the equilibrium phases, are $0.44,0.5$

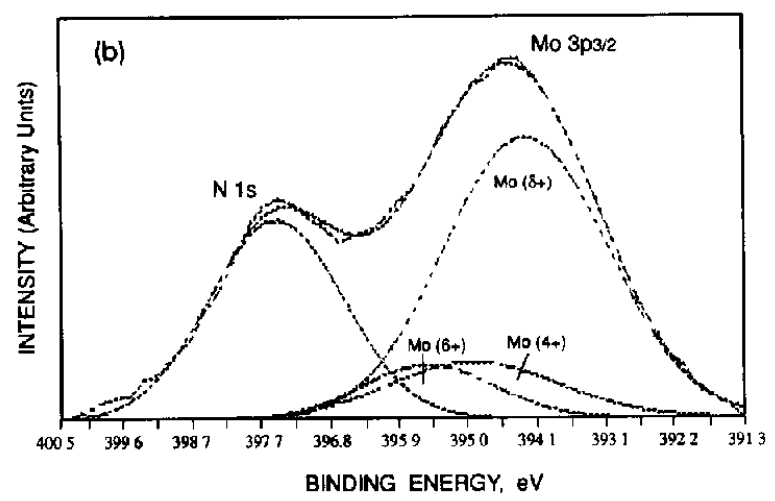

Fig. 3. X-ray photoelectron spectra in the (a) Mo(3d) and (b) Mo(3p) regions. 
and 1.0 , respectively. A small amount of residual oxygen remained in the near-surface region even after reduction of the materials in $\mathrm{H}_{2}$ at $673 \mathrm{~K}$.

\section{Discussion}

Variations in the lattice parameters appeared to be a consequence of variations in the relative amounts of nitrogen and oxygen incorporated into the near-surface region. As the Mo nitrides were prepared using an oxide precursor and since oxygen satisfies the Hägg Rule for Mo compounds (Hägg [12]), its presence in the interstitial sites within the Mo nitride lattice is probable. We should therefore consider the near-surface region for the passivated and reduced materials as being a crystalline oxynitride.

Diffraction patterns arising from regions near the free surface were most consistent with the presence of body-centered lattices. The nearsurface $\mathrm{N} / \mathrm{Mo}$ ratios were characteristic of $\beta$ $\mathrm{Mo}_{16} \mathrm{~N}_{7}$ and /or $\gamma-\mathrm{Mo}_{2} \mathrm{~N}$, or $\delta$-MoN. Although the $\beta-\mathrm{Mo}_{16} \mathrm{~N}_{7}$ phase is body-centered, its lattice parameter is significantly different from those observed experimentally for the molybdenum nitride catalysts. The question remains as to how structures with $\mathrm{N} / \mathrm{Mo}$ ratios (and $(\mathrm{N}+\mathrm{O}$ )/Mo ratios) near unity can give rise to diffraction patterns attributable to body-centered lattices. We have concluded that structures other than those given in the Mo-N phase diagram were present near the surfaces. A plausible structure can be generated, starting with molybdenum metal. The equilibrium crystal structure of molybdenum metal is body-centered cubic (bcc), with a lattice parameter of $0.315 \mathrm{~nm}$ (Villars and Calvert [13]). From atomic radius considerations, nitrogen and oxygen would preferentially occupy the octahedral interstitial sites within the bcc lattice (the cube face centers). As additional nitrogen or oxygen is incorporated into the lattice, more octahedral sites are filled, resulting in a distortion of the unit cell away from a $c / a$ of unity. If all the octahedral interstices are filled, one arrives at a hypothetical primitive cubic $\mathrm{Mo}_{2} \mathrm{~N}_{3-x} \mathrm{O}_{x}$ structure (Fig. 4). This structure can be represented as a five-atom motif consisting of Mo atoms at

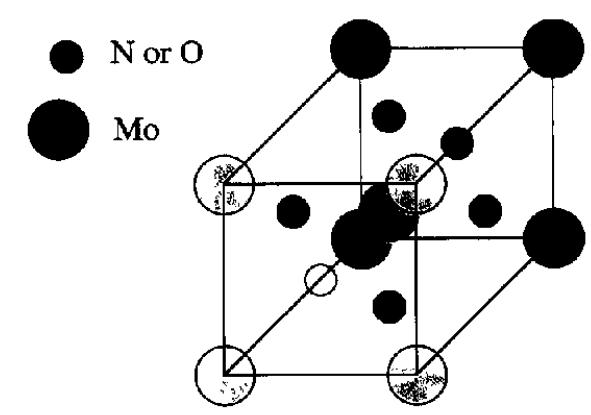

Fig. 4. Hypothetical $\mathrm{Mo}_{2} \mathrm{~N}_{3-x} \mathrm{O}_{x}$ structure in which all of the octahedral interstices are filled (with nitrogen or oxygen). Note: similar shading indıcates atoms lying on the same plane and similar size denotes atoms of the same type

$(0,0,0)$ and $(0.5,0.5,0.5)$, and nitrogen or oxygen atoms at $(0.5,0.5,0),(0.5,0,0.5)$ and $(0,0.5,0.5)$, placed at each corner of a simple cube. This structure can easily reconcile $(\mathrm{N}+$ O)/Mo ratios in excess of unity, as was observed for many of the materials even after reduction in $\mathrm{H}_{2}$. Only half of the octahedral sites are occupied in $\gamma-\mathrm{Mo}_{2} \mathrm{~N}$, the predominant phase found in the bulk (Barrett [14]). It is interesting to note that those materials exhibiting the largest lattice parameters (i.e. those with the largest number of octahedral sites occupied) were also those having the largest $c / a$ ratios, which follows from the above scenario.

Using standard methods (Edington [15]), the structure factor for the hypothetical $\mathrm{Mo}_{2} \mathrm{~N}_{3-x} \mathrm{O}_{x}$ structure was computed. The results for a structure in which all of the octahedral sites are filled are tabulated in Table 3 . In these calculations, atomic form factors of 8.7 for Mo and 2.1 (average of 2.2 and 2.0 for nitrogen and oxygen, re-

Table 3

Structure factor for $\mathrm{Mo}_{2} \mathrm{~N}_{3-x} \mathrm{O}_{x}$

\begin{tabular}{lllc}
\hline$h, k, l$ & $h+k+l$ & $F$ & $I\left(=F^{2}\right)^{\mathrm{a}}$ \\
\hline $\mathrm{U}$ & $\mathrm{E}$ & $2 f_{\mathrm{Mo}}+3 f_{\mathrm{N}, \mathrm{O}}$ & 561.7 \\
$\mathrm{U}$ & $\mathrm{O}$ & $3 f_{\mathrm{N}, \mathrm{O}}$ & 397 \\
$\mathrm{M}$ & $\mathrm{E}$ & $2 f_{\mathrm{Mo}}-f_{\mathrm{N}, \mathrm{O}}$ & 2341 \\
$\mathrm{M}$ & $\mathrm{O}$ & $-f_{\mathrm{N}, \mathrm{O}}$ & 4.4
\end{tabular}

$\mathrm{M}=$ muxed indices; $\mathrm{E}=h+k+l=$ even.

$\mathrm{U}=$ unmixed indices; $\mathrm{O}=h+k+l=$ odd.

${ }^{a}$ Using atomic form factors of 8.7 for Mo and 2.1 (average of nitrogen and oxygen). 
spectively) were used (Edington [16]). This result indicated that the structure factor can, to a first approximation, be represented as that of a bodycentered lattice (Reimer [17]) which is consistent with the diffraction information observed experimentally.

\section{Summary}

We have examined the near-surface structure and composition of a series of molybdenum nitride catalysts via high-resolution transmission electron microscopy and X-ray photoelectron spectroscopy. Fourier analysis of the high-resolution electron micrographs indicated that structures near the surface differed from those in the bulk, most likely due to varying amounts of nitrogen and oxygen in the lattice. Two classes of materials were identified, low-nitrogen-content materials with $(\mathrm{N}+\mathrm{O}) / \mathrm{Mo}=0.73$ and highnitrogen-content materials with $(\mathrm{N}+\mathrm{O}) / \mathrm{Mo}=$ 1.2 (following reduction in $\mathrm{H}_{2}$ at $673 \mathrm{~K}$ ). We concluded that the near-surface structure was that of $\mathrm{Mo}_{2} \mathrm{~N}_{3-x} \mathrm{O}_{x}$, a hypothetical primative cubic structure. This structure accounts not only for the compositions of the materials but also the electron diffraction patterns, which approximate that of a body-centered lattice.

\section{Acknowledgments}

This work was supported by the National Science Foundation under contract NSF CTS9158527, and the Shell Oil Company.

\section{References}

[1] D.J. Sajkowski and S.T Oyama, Am Chem. Soc Div. Pet. Chem. Prepr. 35 (2) (1990) 233.

[2] S.T Oyama, J.C. Schlatter, J.E. Metcalfe and J.M. Lambert, Ind. Eng. Chem. Res. 27 (1988) 1639.

[3] L. Volpe and M.J. Boudart, J. Phys Chem. 90 (1986) 4874.

[4] W.M.H. Sachtler, Faraday Disc. Chem. Soc. 21 (1986) 249.

[5] J. Cowley, Diffraction Physics, 2nd rev. ed. (North-Holland, New York, 1984) pp. 35-49.

[6] J.-G. Choi, J.R. Brenner, C.R. Colling, B.G. Demczyk, J.L. Dunning and L T Thompson, Catal. Today 15 (1992) 201.

[7] W. Rasband, NIH Image, National Bureau of Standards, Research Sciences Branch, NIMH.

[8] A.A. Reeves, Optimızed Fast Hartley Transform for the MC68000 with applications in image processing, MS Thes1s, Thayer School of Engineering, Dartmouth College, Hanover, New Hampshire (1990).

[9] D. Briggs and M.P. Seah, Practical Surface Analysis by Auger and X-Ray Photoelectron Spectroscopy, 2nd ed. (Wlley, New York, 1983) pp. 635-637.

[10] K. Yag1, in: High Resolution Transmission Electron M1croscopy and Associated Techniques, Eds. P. Buseck, J. Cowley and L. Eyring (Oxford Univ. Press, New York, 1988) pp. 577-587.

[11] J.-G. Choi, C W. Colling and L.T Thompson, in preparation.

[12] G Hagg, Z. Phys. Chem. 12 (1931) 339.

[13] P. Villars and L.D. Calvert, Pearson's Handbook of Crystallographic Data for Intermetallic Phases, 2nd ed., Vol. 4 (ASM International, Metals Park, OH, 1991) p. 4407.

[14] C.S. Barrett, Structure of Metals: Crystallographic Methods, Principles and Data, 3rd rev. ed. (Pergamon, New York, 1980) pp. 301-303.

[15] J.W. Edıngton, Practical Electron Microscopy in Materials Science (Philıps, Eindhoven, 1976) pp. 36-39.

[16] Ref. [15], pp. 321-322.

[17] L. Reimer, Transmission Electron Microscopy: Physics of Image Formation and Microanalysis (Springer, New York, 1984) pp. 272-277 